\title{
The use of degradable nerve conduits for human nerve repair: A review of the literature
}

doi:10.1533/abbi.2004.0008

\author{
M. F. Meek, K. Jansen and P. H. Robinson \\ Department of Plastic Surgery, University Medical Center Groningen, Hanzeplein 1, 9700 RB Groningen, \\ The Netherlands
}

\begin{abstract}
The management of peripheral nerve injury continues to be a major clinical challenge. The most widely used technique for bridging defects in peripheral nerves is the use of autologous nerve grafts. This technique, however, has some disadvantages. Many alternative experimental techniques have thus been developed, such as degradable nerve conduits. Degradable nerve guides have been extensively studied in animal experimental studies. However, the repair of human nerves by degradable nerve conduits has been limited to only a few clinical studies. In this paper, an overview of the available international published literature on degradable nerve conduits for bridging human peripheral nerve defects is presented for literature available until 2004. Also, the philosophy on the use of nerve guides and nerve grafts is given.
\end{abstract}

Key words: Nerve repair, degradable, nerve guide, human, peripheral nerve.

\section{AUTOLOGOUS NERVE GRAFTING}

Despite recent advances in the understanding of nerve regeneration and microsurgical techniques, the clinical outcome of peripheral nerve injuries remains disappointing (Lundborg 2000). When the length of the gap created by substance loss is too large to permit direct suturing without tension, nerve grafts are usually employed. The sural nerve (a sensory nerve) from the calf is the most commonly used as an autologous nerve graft. Other suitable donor sites are available such as the lateral and medial antebrachial cutaneous nerves from the forearm. Morbidity at the acceptor and donor sites should be considered when autologous nerve grafts are used for peripheral nerve reconstruction which, however, is generally in practise and relatively minor.

A common problem is that the fascicular pattern (the number as well as the diameter of the fascicles) of the nerve ends rarely corresponds to the fascicular pattern of the nerve graft. Slow and/or incomplete reinnervation of the target organ are often the consequences partly due to cross-innervation. Nerve grafting complicates nerve regeneration by introducing two suture lines, with the possibility of neuroma-in-continuity formation, which may

Corresponding Author:

Marcel F Meek

Department of Plastic Surgery, University Medical Center Groningen

Hanzeplein 1, 9700 RB Groningen, The Netherlands

Tel: +31-50-3613531 Fax: +31-50-3613041

Email: marcelfmeek@hotmail.com lead to painful sensations and poor recovery of nerve function. Another disadvantage of two suture lines is that more suture material is necessary which in turn causes more intraneural fibrosis. Fibrosis negatively influences the quality of nerve regeneration. Scar tissue formation occurs in the nerve graft. The effects are most obvious at the distal suture line, because this is the last obstacle to be overcome by the regenerating axons. When this occurs, revision of the distal suture line should be performed, which means a second operation. The axon sprouts may also unavoidably divert into extraneural tissue (Meek 2000).

As mentioned previously, donor site morbidity as well as the limitation in the amount of tissue available has to be considered. Donor site morbidity may involve: loss of donor nerve function, hyperesthesia adjacent to and sometimes in the area of sensory loss, painful neuroma formation at the site of the division, and unpleasant sensations after minor trauma to this area even when the neuroma is deeply embedded in surrounding tissue.

\section{NERVE CONDUITS}

During the last decades, much research has focused upon the experimental nonhuman application of nerve conduits in peripheral nerve repair. The concept behind the use of a nerve guide (also known as nerve guidance channel or nerve conduit) is that regenerating nerve fibers are allowed to grow toward the distal nerve stump, while the necessity of a human donor site is prevented. A nerve graft guide can meet many of the needs of peripheral nerve reconstruction by isolating the local regenerative 
environment, thereby leading to an increase in the concentration of neurotropic and neurotrophic factors produced by the damaged nerve stumps, and by reducing fibroblast invasion and subsequent scarring of the nerve. In addition, it provides directional guidance to the regenerating axons and prevents neuroma formation and excessive branching at the repair site. Care should be taken with regard to the tube dimensions (the inner diameter and wall thickness of the nerve conduit in relation to the diameter of the nerve) (Den Dunnen 1996; Meek 2000). A nerve guide should have an internal diameter large enough to overcome problems when telescoping the proximal and distal nerve stumps into the lumen of the nerve guide during the implantation procedure, and should have a thin wall that swells minimally during degradation and causes no nerve compression. Tubes with thin walls have been shown to be associated with less neuroma formation proximal and distal to the tube because of greater elasticity of thin-walled tubes compared to thick-walled tubes. However, a guide with a too large internal diameter might stimulate fibrous tissue ingrowth into the lumen of the nerve guide, and if the wall is too thin this may cause collapse of the nerve guide, thereby hampering nerve regeneration and maturation. Several materials, either of biological origin (e.g. veins and muscle) or synthetically fabricated (both biodurable and degradable), were used in clinical trials, with varying success (Meek 2002). For example, Merle et al (1989) used nerve guides composed of silicon rubber. Two years after reconstruction, the patients, however, still complained of loss of nerve function and in these situations a second operation was necessary. The disadvantage of nondegradable nerve guides is that the material remains in situ as a foreign body, potentially causing a chronic foreign body reaction with excessive scar tissue formation, possibly resulting in constriction of the regenerating nerve, ultimately limiting recovery of nerve function (e.g. secondary nerve impairment) (Merle 1989). Some years ago an overview of the use of nerve conduits in peripheral nerve conduits was described by Meek and Coert (2002); recent clinical investigators that previously focused upon nondegradable nerve conduits, now also advise the use of degradable conduits for peripheral nerve repair (Lundborg 2004).

\section{DEGRADABLE NERVE CONDUITS}

The concept of degradable nerve guides is that the nerve guide directs the outgrowing nerve fibers toward the distal nerve stump, while preventing neuroma formation and ingrowth of fibrous tissue into the nerve gap. The nerve guide gradually degrades with a minor foreign body reaction and therefore is a potential alternative for nondegradable nerve conduits.

Degradable nerve conduits have been widely used as conduit material in experimental nerve regeneration studies. However, the number of published clinical studies using degradable nerve conduits in the repair of peripheral nerve defects is very limited. An overview of the literature on the clinical use of degradable nerve conduits in human peripheral nerve repair is presented below for literature available until 2004.

\section{History}

Despite the fact that many researchers think degradable materials were only used in the last decades, earlier articles report the opposite. According to Professor MC Vanlair, from Liège, Gluck, proposed the idea to use the decalcified bone tubes in 1881 (Vanlair 1882). He used the central canal of a decalcified bone as a conduit between the nerve stumps. Payr, in 1900, used magnesium conduits in the human median, peroneal and vagus nerves. In 1901, Lotheissen published that the disadvantage of Vanlair and Payr's techniques was the quick resorption of the nerve conduits. The latter also caused sharp edges during degradation potentially causing damage to nerve tissue. Lotheisen himself used gelatine as conduit material in humans. However, the nerves that were reconstructed were not mentioned. Foramitti, in 1904, used gelatine conduits twice for radial nerve repair. Objective measurement techniques to evaluate functional nerve recovery after bridging the nerve defects is not given in any of the papers.

\section{Polyglycolic acid (PGA) nerve conduit}

Mackinnon and Dellon (1990) described 15 patients in a prospective study of secondary nerve reconstructions of digital nerves. Excellent sensation was found in 33\% and good sensation in 53\%. Poor recovery was seen in 14\%. They were the first to describe the utilization of a sensory reeducation program for their patients. In defects up to $30 \mathrm{~mm}$, the PGA tubes produced at least comparable results to the classic nerve graft.

Crawley and Dellon (1992) used a PGA conduit to reconstruct a $25-\mathrm{mm}$ defect in the right inferior alveolar nerve. A 51-year-old woman developed immediate and persistent numbness of the right lower lip and chin with no associated dysesthesia after extraction of a right mandibular molar tooth. The proximal end of the inferior alveolar nerve was sutured into the polyglycolic acid tube, and the mental nerve was sutured into the distal end of the tube. Two years following nerve reconstruction, pain relief remained excellent and perception of pressure and vibration was similar to the thresholds for these perceptions on the contralateral lip.

Weber et al (2000) described 136 nerve transections in the hand. These were randomized into two groups, standard repair or repair using a PGA tube. Sixtytwo patients were treated with a PGA tube. This is the only prospective, randomized multicenter study on biodegradable tubes: $78 \%$ were primary repairs, $14 \%$ delayed, and $8 \%$ secondary. The sensory nerve defects distal to the wrist crease were up to $30 \mathrm{~mm}$ with an 
average of $7 \mathrm{~mm}$. The follow-up was 12 months, so a further improvement of nerve function may be expected. All patients received sensory reeducation. Additional procedures were repair of tendon and bony lacerations. In nine patients a delayed primary amputation was performed. In two patients the tubes extruded. The conduit repair gave significantly better results than the standard end-to-end repair. Excellent results were obtained in 91\% in the PGA group ( $3.7 \mathrm{~mm}$ moving two-point discrimination), whereas in the end-to-end group only $49 \%$ achieved excellent results $(6.1 \mathrm{~mm}$ moving two-point discrimination). In defects over $8 \mathrm{~mm}$ the conduit showed significantly superior results.

Casañas et al (2000) described a prospective study of 17 patients with chronic lesions of the digital nerves of the hand, and lesion defects longer than $2 \mathrm{~cm}$. These defects were repaired by PGA tubes. They concluded that the use of PGA tubes was a useful technique for repairing chronic sensory nerve lesions or defects longer than $2 \mathrm{~cm}$. Sensory reeducation was not used.

Recently, Kim and Dellon (2001) used a polyglycolic acid nerve conduit for reconstruction of a 2 -cm nerve defect after post-traumatic neuroma resection of the medial plantar nerve in the foot. Secondary digital nerve reconstruction using a degradable nerve conduit had not been previously reported, and illustrated successful reconstruction.

Hagiwara et al (2002) used a PGA tube containing a collagen sponge for the reconstruction of an intrapelvic nerve that was sacrificed during extensive resection of recurrent intrapelvic rectal cancer. Return of nerve function was obtained after 4 months. For us, it is unclear whether this tube filled with a sponge was FDA approved or not.

A PGA nerve conduit, called the Neurotube ${ }^{\mathrm{TM}}$, is produced by Neuroregen and was FDA approved for human use in 1999. In the Netherlands the conduit can be bought for approximately 350 Euro. The nerve conduit is corrugated to resist occlusive forces of the surrounding soft tissue, and is made from sugars and amino acids that are naturally found in the body. The nerve conduit is absorbed in the body by means of hydrolysis within 90 days of implantation (Barrows 1986; Ginde 1987). Negative aspects are the limitation in gap length that can be bridged and the material is relatively hard which makes suturing difficult. However, this design does withstand pressure, even permitting joint flexion without kinking. Polyglycolic acid material is also currently used in absorbable sutures, and in mesh form to wrap internal organs injured following trauma.

\section{Poly(DL-lactide- $\varepsilon$-caprolactone) nerve conduit}

In the 1980s, the Department of Plastic Surgery, University Hospital Groningen, the Netherlands, in cooperation with the Department of Polymer Chemistry at the Groningen State University, the Netherlands, developed a nerve guide composed of a copolymer of poly DL-lactic acid and poly $\varepsilon$-caprolactone [p(DLLA- $\varepsilon-C L)]$. In the animal setting, the nerve guide degraded completely within 1 year, showed only a mild foreign body reaction, and fast nerve regeneration (Den Dunnen 1996; Meek 2000). However, despite our previous experimental reports, some very small fragments of biomaterial were still found on the outside of the perineurium of the regenerated nerve after 16 months of implantation, indicating a minor secondary foreign body reaction (Jansen 2004). To understand the discrepancy between the experimental results after 12 and 16 months of implantation, it should be stated that in the previous studies cross-sections for LM and EM work were performed to evaluate the degradation behavior, while more recently also transverse-sections over the whole length of the tube including the nerve were performed (Jansen 2004). In these preparations we can still see biomaterial fragments at 16 months after implantation. This was an unexpected finding.

Poly(DLLA- $\varepsilon-C L)$ nerve guides are clinically being tested in the hand in a multicenter trial and the first preliminary results were published by Meek et al (2003). Conventional suturing as well as autologous nerve grafting are acceptable methods in the control group within the protocol of the study. Nerve guides with various diameters were used for different levels of nerve repair. In 2003, it was shown that the operation was technically successful in the first three nerve conduits in two patients. The first patient had a $5-\mathrm{mm}$ defect in the ulnovolar digital nerve of the thumb. The second patient had a $12-\mathrm{mm}$ defect in the ulnovolar nerve of the little finger and an $8-\mathrm{mm}$ defect in the common digital nerve of the fourth and little fingers. In June 2003, the preliminary results of the first 13 patients were presented (Bertleff 2003), while now 54 patients have been included in the study. Functional nerve recovery will be assessed with a 1 year follow-up. At the moment of writing this review, the study is almost finished and a final report will be published by the first author of this paper. A sensory reeducation program is not followed in these patients.

Negative aspects of the $p(D L L A-\varepsilon-C L)$ nerve guide are the limitation in gap length that can be bridged and the relative high cost as the product is now being commercialized. In the Netherlands the tube is available for approximately 650 Euro. It is not known how the minor secondary foreign body reaction that was found recently in the experimental setting will "behave" in the human situation. It would be interesting to evaluate the complications in terms of wound problems, infections, and protrusion of the conduit. The material is flexible, which makes implantation of the tube easy. However, problems may be expected to arise if the tube is subjected to compression or if the tube is placed over a joint with the risk of kinking. The tube may then easily collapse and return of sensibility will not occur. Further reports will tell whether this will happen or not. It is advised to put the tube in warm water before implantation to facilitate easy suturing. This nerve conduit is also FDA approved. 


\section{DISCUSSION}

The nerve guide has served its purpose after the nerve fibers have bridged the gap between the proximal and distal nerve stumps, and its presence in the case of a nondegradable material may even have a negative influence on the regenerated nerves. Therefore, nerve guides should preferably be made of biodegradable materials. Nerve guides should degrade as soon as possible after nerve regeneration. Fine tuning of the degradation of a nerve guide is extremely important for the final result after the nerve reconstruction. The degradation rate of the nerve guide should be synchronized to the axonal growth rates. Nerve guides should be made of a biocompatible material. It should be non-cytotoxic, non-carcinogenic, nonimmunogenic, non-mutagenic, and should not cause any irritation or local or systemic allergic response. The biological safety of the various ingredients used to manufacture the nerve guide, the potential leachable substances or degradation products, and the final composition of the biomaterial should therefore be guaranteed. Furthermore, a nerve guide should be flexible and transparent, which allows accurate observation of the nerve stumps when telescoping them into the nerve guides, but must not impede neural regeneration because of kinking or collapse of the tube. Thus far, there have been only a limited number of degradable nerve conduits used in the clinical setting, despite many animal experimental studies that have been performed.

Complications after implantation of a degradable biomaterial may include infection, fistula, exposure, extrusion, displacement, implant fracture, seroma, edema, prominence, pain, inflammatory reaction, and hypersensitivity. For instance, care should be taken to avoid tension when implanting foreign bodies under a soft-tissue cover, because of the risk of extrusion of the implant. Pain and prominence are often due to technical factors. We agree with Dahlin and Lundborg (2001) that degradable tubes may seem ideal, but they may introduce new problems associated with the resorption process in terms of a substantial unrestricted macrophage invasion, fibrosis, and disorganized axonal growth, especially in combined (motor and sensory) nerves (e.g. median nerve). The company selling the conduit materials should ensure safety of the device and the factors that may contribute to morbidity should be minimized.

Several authors using the same biomaterial may have different outcomes in the future because of different implant preparation techniques, perioperative antibiotic regimes, operative techniques, and compositions methods of conduit fixation and slight differences in biomaterial. Therefore, when changes are made in standard protocol like the exact composition of biomaterial(s), new extensive biocompatibility studies (including long-term degradation studies) should be performed before clinical application in order to guarantee the biological safety of the product.

\section{CONCLUSION}

The clinical application of degradable nerve conduits for nerve defects larger than 3 or $4 \mathrm{~cm}$ is not much further than it was around 1900, despite the increase in experimental knowledge about nerve regeneration and bridging nerve gaps. Therefore, the use of autologous nerve grafts is still the golden standard for nerve defects of more than $3 \mathrm{~cm}$. Nowadays, a nerve conduit can be used in case of peripheral nerve defects up to $3 \mathrm{~cm}$ based upon the degradation rate of these tubes.

When considering the aquisition of one of the presently available conduits one may consider that the advantages of the Neurolac have not yet been demonstrated in comparison to the much cheaper Neurotube. The fact that long-term outcomes of a prospective multicenter study of the Neurotube is available and the relatively high costs 650 Euro for the Neurolac versus 350 Euro for the Neurotube in the Netherlands, we would suggest that the Neurotube be used for peripheral nerve repair up to $3 \mathrm{~cm}$ with appropriate nerve diameters. Furthermore, we advise additional degradation studies of the $p(D L L A-\varepsilon-C L)$ nerve guide and careful analysis of possible complications that may occur with nerve conduits because of the recent findings of Jansen et al (2004).

Different degradable materials will probably be available in the next few years. Important for the peripheral nerve surgeon is that the company that sells the conduits should be able to provide results on the long-term degradability (>1 year).

Because of an expected increase in the number of papers on nerve conduits in the forthcoming years, it would be of benefit for the peripheral nerve surgeon to have a summary of the available FDA- and CE-approved nerve conduits that are on the market in order to know what tubes are available and to be better able to choose between the different tubes. New tubes that claim to be degradable should show their advantages compared to the presently available tubes with regard to the quality of nerve regeneration and cost.

\section{REFERENCES}

Barrows TH. 1986. Degradable implant materials: A review of synthetic absorbable polymers and their applications. Clin Mater, 1:233.

Bertleff M JOE, Meek MF, Robinson PH et al. 2003. Introduction NEUROLAC ${ }^{\circledR}$ trial and the preliminary results. Presented at the IInd congress of the WSRM, Heidelberg, Germany. 11-14 June 2003.

Büngner O von. 1891. Ueber die Degeneration und Regenerationsvorgänge am Nerven nachVerletzungen. Beitrage zur Pathologische Anatomie und zur Pathologie, 10:321-393 + Tafel XVI-XVII.

Casañas J, Serra J, Orduna M et al. 2000. Repair of digital sensory nerves of the hand using polyglycolic acid conduits. 7 Hand Surg [Br] 25B:44. 
Crawley WA, Dellon AL. 1992. Inferior alveolar nerve reconstruction with a polyglycolic acid bioabsorbable nerve conduit. Plast Reconstr Surg, 90:300-302.

Dahlin LB, Lundborg G. 2001. Use of tubesin peripheral nerve repair. Neuros Clin N Am, 1;12:341-352.

Den Dunnen WFA. 1996. Biodegradable nerve guides. The Netherlands: University of Groningen. ISBN: 90-9009659-0.

Foramitti C. 1904. Zur Technik der Nervennaht. Arch Klin Chir 73:643.

Ginde RM, Gupta RK. 1987. In vitro chemical degradation of polyglycolic acid pellets and fibers. F Appl Polym Sci 33:2411.

Hagiwara A, Nakashima S, Itoh T et al. 2002. Clinical application of PGA-tube for regeneration of intrapelvic nerves during extended surgery for intrapelvic recurrent rectal cancer. Gan To Kagaku Ryoho, 29:2202-2204.

Jansen K, Meek MF, van der Werff JFA et al. 2004. Long-term regeneration of the rat sciatic nerve through a biodegradable poly(DLLA- $\varepsilon-C L)$ nerve guide: Tissue reactions with focus on collagen III/IV reformation. $\mathcal{7}$ Biomed Mater Res, 69A:334-341.

Kim J, Dellon AL. 2001. Reconstruction of a painful post-traumatic medial plantar neuroma with a bioabsorbable nerve conduit: A case report. 7 Foot Ankle Surg, 40:318-323.

Lotheissen G. 1901. Zur Technik der Nerven-und Sehnennaht. Arch Klin Chir, 64:310-313.

Lundborg G. 2000. A 25-year perspective of peripheral nerve surgery: Evolving neuroscientific concepts and clinical significance. 7 Hand Surg, 25A:391.
Lundborg G, Rosen B, Dahlin L et al. 2004. Tubular repair of the median or ulnar nerve in the human forearm: A 5-year follow-up. 7 Hand Surg, 29A:100-107.

Mackinnon SE, Dellon AL. 1990. Clinical nerve reconstruction with a bioabsorbable polyglycolic acid tube. Plast Reconstr Surg, 85:419-424.

Meek MF. 2000. Artificial Nerve Guides-assessment of nerve function. The Netherlands: University of Groningen. ISBN: 90-3671303-x.

Meek MF, Coert J H. 2002. Clinical use of nerve conduits in peripheral-nerve repair: Review of the literature. 7 Reconstr Microsurg, 18:97-109.

Meek MF, Bertleff MJ, Ritt MJ et al. 2003. A degradable artificial nerve guide to bridge peripheral nerve defects. Ned Tijdschr Geneeskd, 147:717-721.

Merle M, Dellon AL, Campbell JN et al. 1989. Complications from silicon-polymer intubulation of nerves. Microsurgery, 10:130-133.

Payr E. 1900. Beiträge zur Technik der Blutgefäss- und Nervennaht nebst Mitteilungen über die Verwendung eines resorbierbaren Metalles in der Chirurgie. Arch Klin Chir, 63:67-93.

Vanlair C. 1882. De la regeneration des nerfs peripheriques par le procede de la suture tubulair. CR Acad Sci (Paris) 65: 99-101.

Weber RA, Breidenbach WC, Brown RE et al. 2000. A randomized prospective study of polyglycolic acid conduits for digital nerve reconstruction in humans. Plas Reconstr Surg, 106:1036-1045. 

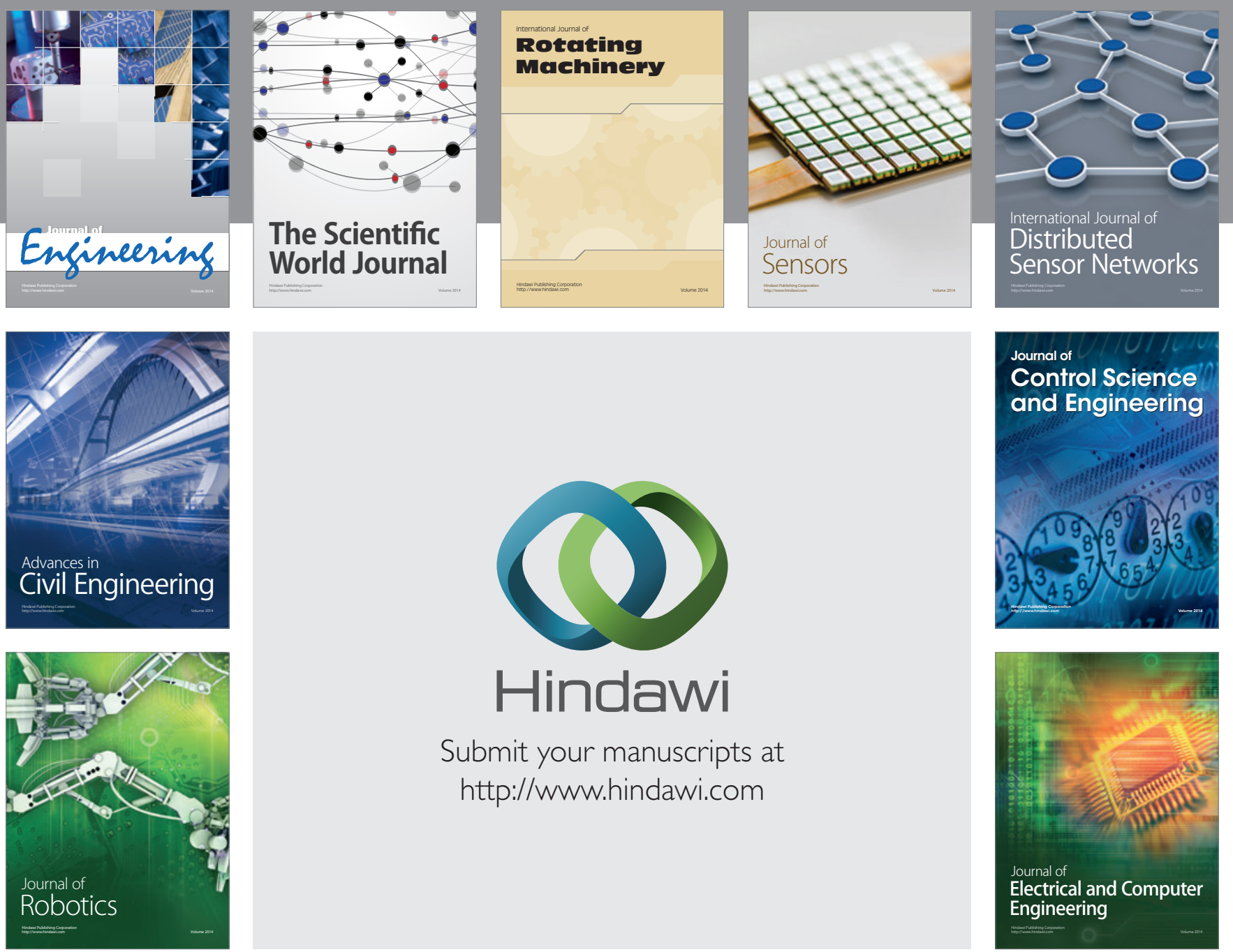

Submit your manuscripts at

http://www.hindawi.com
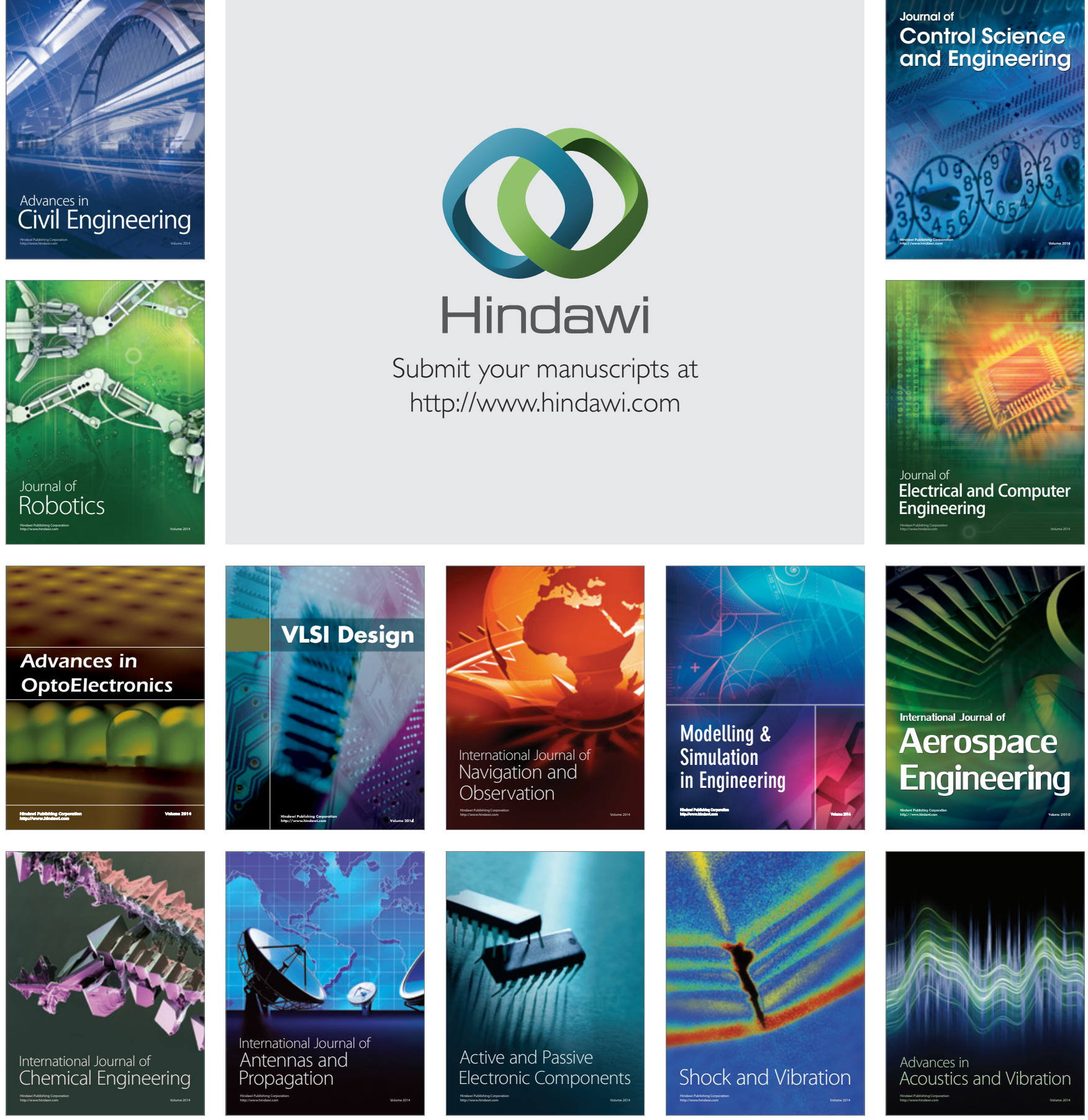